\title{
Points to ponder in the study of cadmium iodide
}

\author{
Harjeet Kaur \\ Department of Physics, Maharshi Dayanand University, Rohtak - 124001, India \\ Tel: 09416351115 \\ E-mail address: sunilkc2001in@yahoo.com
}

\begin{abstract}
During the growth of cadmium iodide crystals from solution, it has been observed that sometimes the crystal suddenly rotates. Probably, the convection currents are providing the force for the rotation. Mathematically, this force can be estimated and it must be greater than the van-der Walls force acting between adjacent sandwiches of cadmium iodide and can give rise to polytypic phase transformations. Further, studies of vacancies and the density measurements of various polytypes can help in understanding the phenomenon of polytypism better.
\end{abstract}

Keywords: cadmium iodide; lead iodide; polytypism; phase transformations; Inorganic crystal structure

\section{INTRODUCTION}

Lot of work has been done on the polytypism of cadmium iodide and lead iodide. The research work on polytypism has been mainly confined to the structure determination of the crystals and till date more than 200 structures have been determined and number of theories put forward from time to time but the problem of polytypism is yet to be understood [1]. After such a long time of experimental work, one is not in a position to grow crystals of one's choice. Recently, Kasi et.al. [2] has reported the fabrication and characterization of $\mathrm{PbI}_{2}$ nanocrystals. They have reported $2 \mathrm{H}$ polytype and some presence of $4 \mathrm{H}$. It so appears that $4 \mathrm{H}$ might be confused with $12 \mathrm{R}$ as Chaudhary and Trigunayat [3] have widely studied the subject and reported 12R as a dominant structure in melt growth. Similarly Flahaut et. al. [4] have also reported more $2 \mathrm{H}$ polytypes in carbon nano tubes than $4 \mathrm{H}$ polytype. No higher polytypes have been reported. In most of the studies, $\mathrm{CdI}_{2}$ crystals have been mainly grown from solution. When the crystals are grown from solution, the growth rate is quite high and there is random motion of molecules and statistically there is every possibility of formation of higher and disordered polytypes. That is the reason why maximum number of polytypes have been observed in case of solution grown cadmium iodide crystals. In this paper, it has been tried to peep into the factors that have been overlooked or not reported as far as polytypism of cadmium iodide is concerned. 


\section{BASIC STRUCTURE OF CdI 2}

The $\mathrm{CdI}_{2}$ structure consists of various stackings of $\mathrm{CdI}_{2}$ sandwiches in each of which a layer of $\mathrm{Cd}$ ions is sandwiched between two close packed layers of iodine ions. I-Cd-I sandwich being the repeat unit and each $\mathrm{Cd}$ atom is surrounded by $6 \mathrm{I}$ atoms forming a near octahedral $\left[\mathrm{CdI}_{6}\right]^{4-}$. The interlayer interactions are more covalent than ionic and the electron density between the sandwiches is very low that results in weak van der-Walls interactions between the sandwiches [5] of the type:

$$
U(R)=-d / R^{6}+b e^{-a R}
$$

$\mathrm{R}$ is the separation between the atoms.

$\mathrm{a}, \mathrm{b}$ and $\mathrm{d}$ are constants characteristics of atoms.

In the case of $\mathrm{CdI}_{2}$, the relative bond strengths between iodine atoms within a sandwich and iodine atoms between adjacent sandwich have been estimated around 125 [6]. This results in many polytypes and complicated phase changes amongst different polytypes. As ICd-I sandwich contains three layers of atoms and charge carrier dynamics perpendicular to these layers have several quantum states. This is in contrast to the two dimensional graphene where charge carrier dynamics involving direction perpendicular to the layers of carbon atoms can be pinned to the ground state.

\section{MACROSCOPIC OBSERVATIONS}

It has been observed that while growing crystals from solution, in some cases few thin crystals suddenly rotate in their own plane. Motion is in fact pure rotation. This rotation is due to the convection currents in the solution. Since the crystals are hexagons, their angle of rotation can be estimated to a fair approximation. On can make an estimate of the force acting on the crystal due to convection current as follows (Fig. 1). If $\mathrm{m}$ is mass of the crystal and $\mathrm{F}$ is the force acting on it. If force rotates the crystal through $\Theta$ then one can safely write

$$
\mathbf{F . r}=\mathrm{Id} \mathrm{d}^{2} \Theta / \mathrm{dt}^{2}
$$

Integrating the above equation twice and considering the hexagonal crystal to be a circle i.e. moment of inertia

$$
\mathrm{I}=1 / 2 \mathrm{mr}^{2}
$$

One can simply arrive at

$$
\mathbf{F}=\mathrm{mr} \Theta / \mathrm{t}^{2}
$$

Further

$$
\mathbf{F}=\pi r^{2} \mu \rho \Theta / t^{2}
$$


Where symbols $\mu$ and $\rho$ stand for thickness and density of the crystal. This force is tangential in nature and Force acing per unit area acting per unit area of the crystal.

$$
\mathrm{F} / \pi r^{2}=\mu \rho \theta / \mathrm{t}^{2}
$$

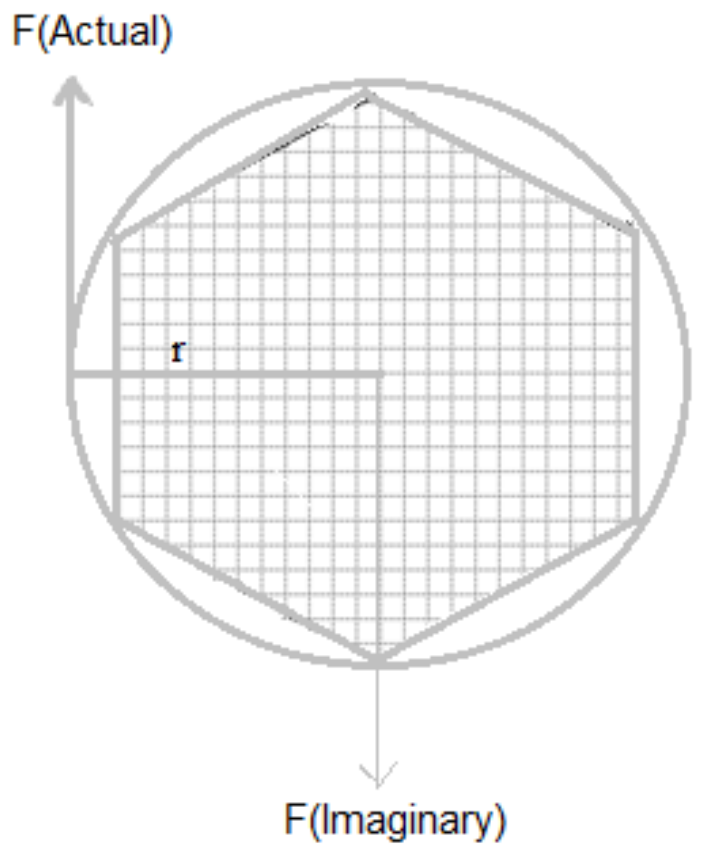

Fig. 1. Line sketch of hexagonal cadmium iodide crystal.

Trigunayat [7] has calculated the value of force acting per unit area on a typical crystal to be of the order $1 \mathrm{gm} \mathrm{mm}^{-2}$ and have qualitative shown that value of critical shear stress required to create dislocation in cadmium iodide crystal is less than $1 \mathrm{gm} \mathrm{mm}^{-2}$.

The dislocation velocity in a material is usually related by net shear stress acting on the dislocation $\sigma_{\mathrm{d}}$ by the equation $\mathrm{V}_{\mathrm{SS}} \alpha \sigma_{\mathrm{d}}{ }^{\mathrm{m}}$ where $\mathrm{m}$ varies between 5-40 for metals but in ionic compounds like $\mathrm{CdI}_{2}$ the dislocation velocity is proportional to the stress i.e. $\mathrm{m}=1$ [8]. From the above fact it is clear that in case of $\mathrm{CdI}_{2}$ stored for longer times stresses available are due to impurities (of the order of few ppm) which are very small and the phase transformations amongst polytypes takes as long as many years to take place [1].

In addition to other factors, the rotation of sandwiches due to convection currents that adds to give rise to phase transformations in the crystals but has never been considered.

This fact has not been considered by Wahab and Trigunayat [5] that speaks about theoretical work on the rotation and translations of sandwiches for the phase transformation amongst polytypes, nor by Wahab and Kant's model [6] that suggests the simultaneous occurrence of basic phases $2 \mathrm{H}$ and $4 \mathrm{H}$ in solution growth under similar growth conditions and higher polytypes are formed by various combinations of the above two structures.

Mathematically it has been expressed as: 


$$
\mathrm{NH}=\mathrm{n}_{1} \times 2 \mathrm{H}+\mathrm{n}_{2} \times 4 \mathrm{H}+-------
$$

Where $\mathrm{N}$ is number of atomic layers in the unit cell of polytype.

$\mathrm{n}_{1}, \mathrm{n}_{2}$ and $\mathrm{n}_{3}$ are number of units of basic phases.

\section{MICROSCOPIC OBSERVATIONS}

Let us consider the simplest case of $4 \mathrm{H}$ structure. From geometric perspective the structure can be expressed as

$$
\mathrm{A} \beta \mathrm{C} \text { BaC........ }
$$

It can alternately be expressed as $\mathrm{A} \beta \mathrm{C} \mathrm{A} \gamma \mathrm{B} \ldots \ldots . .$.

Similarly it can be expressed as AYB CaB........ and so on.

A total of 12 equivalent geometrical possible structure are there. Similarly, one can consider the structure $6 \mathrm{H}$ and it will have more no of possible structures.

Further, the structure reported as $4 \mathrm{H}$ may be a mixture of the above equivalent sequences. But X-ray diffraction is not sensitive enough to detect the above difference. In addition, it is yet to be discovered if $4 \mathrm{H}$ transforms into $4 \mathrm{H}$ with different stackings. Topography can be the best tool for such studies in which one can even observe the dislocations. It has been observed that in most of the cases of studies of X-ray diffraction of cadmium iodide crystals only a particular small area equal to spot size of X-rays is studied in the structure work. But whole area of the crystal is not scanned, again the best way out is the topography.

\section{VACANCIES AND DENSITY MEASUREMENT}

In most of the earlier studies made on $\mathrm{CdI}_{2}, \mathrm{PbI}_{2}$ and other polytypic crystals, the role played by the vacancies is being ignored. It is a convincing fact that an atom surrounded by vacancies is more mobile in the layer plane and even in a direction perpendicular to it.

The degree and the distribution of vacancies is also an important parameter to be looked for the understanding of polytypism and fabrication of devices from polytypic crystals. Recently Chaudhary [9] has reported melt grown crystals of $\mathrm{PbI}_{2}$ are best suited for the nuclear radiation detectors. Further, Ito et. al [10] have estimated that with $\mathrm{Si}$ vacancy in $\mathrm{SiC}$ polytype $6 \mathrm{H}$ structure is formed and $4 \mathrm{H}$ is formed in $\mathrm{C}$ vacancy condition. Similar calculations have been done on $\mathrm{ZnS}$ crystals [11].

\section{CONCLUSION}

It is suggested that with the advance and sensitive instrumentation available, the density measurement of various polytypes can give clue about the vacancies and their distribution, which in turn can help in understanding the phenomenon of polytypism Some experimental work is also being done on the similar polytypic crystals $\mathrm{PbI}_{2}$ [12]. 


\section{References}

[1] G. C. Trigunayat, Phase Trans 16/17 (1989) 509.

[2] G. K. Kasi, R. D. Norman, S. Ahmadi J. Phys. D. Appl. Phys. 40 (2007) 1778.

[3] S. K. Chaudhary, G. C. Trigunayat, Cryst. Res. Technol. 23 (1988) 889.

[4] E. Flahaut, J. Sloan, et.al. Chem. Mater. 18 (2006) 2059.

[5] M. A. Wahab, G. C. Trigunayat, Solid State Communications 36 (1981) 885.

[6] M. A. Wahab, R. Kant, Crys. Res. Technol. 22 (1986) 239.

[7] G. C. Trigunayat, Z. Fur. Kristall. 122 (1965) 463.

[8] M. F. Kanninen, A. R. Rosenfield, Phil. Mag. 20 (1969) 569.

[9] S. K. Chaudhary, Crystal Structure Theory and Applications 1 (2012) 21.

[10] T. Ito, T. Kondo, T. Akiyama, K. Nakamura, Journal of crystal growth 318 (2011) 141.

[11] T. Ito, T. Kondo, T. Akiyama, K. Nakamura, Phys. Stat. Solidi (c) 8(2) (2011) 583.

[12] D. S. Bhavsar, Archieves of Physics Research 3(2) (2011) 146. 